\title{
A $200 \mathrm{GHz}$ Downconverter in 90nm CMOS
}

\author{
Maarten Tytgat, Michiel Steyaert and Patrick Reynaert \\ K.U. Leuven ESAT-MICAS \\ Kasteelpark Arenberg 10 \\ B-3001 Leuven, Belgium \\ maarten.tytgat@esat.kuleuven.be
}

\begin{abstract}
A $200 \mathrm{GHz}$ downconverter in $90 \mathrm{~nm}$ standard CMOS is presented with a measured positive conversion gain of $+6.6 \mathrm{~dB}$ and an IF bandwidth of $3 \mathrm{GHz}$ for an LO power of $-14.9 \mathrm{dBm}$. The conversion gain has a flatness of $\pm 1.5 \mathrm{~dB}$ in an $\mathrm{LO}$ frequency range of $26 \mathrm{GHz}$. The $\mathrm{IIP}_{3}$ is $-5.4 \mathrm{dBm}$. BPSK and QPSK data downconversion are demonstrated with a data rate of over $4 \mathrm{Gbit} / \mathrm{s}$.
\end{abstract}

\section{INTRODUCTION}

As CMOS scaling continues, new opportunities arise for electronic integrated circuits working at ever higher frequencies. The millimeter wave frequency range, including the G-band from 140 to $220 \mathrm{GHz}$, allows applications such as high speed short range communication, imaging and radar. In this paper, the design and measurements of a $200 \mathrm{GHz}$ downconverter are discussed. The LO frequency $\left(f_{\mathrm{LO}}\right)$ of the mixer is $200 \mathrm{GHz}$, which is well above the $f_{T}(144 \mathrm{GHz})$ of the used technology. The IF output has a bandwidth of several $\mathrm{GHz}$ To the author's knowledge, this is the first integrated down conversion mixer in CMOS above $200 \mathrm{GHz}$ with demonstrated capability of Gbit/s data communication.

\section{CiRcuit DESIGN AND IMPLEMENTATION}

Fig. 1 shows the architecture of the complete downconverter chip. The circuit can be divided into two parts, according to their operating frequency: the RF part and the IF part. The former consists of the RF and LO transformers and the four mixing transistors. The latter comprises a transimpedance amplifier (TIA), voltage gain stage and voltage buffer.

The four mixing transistors, together with the TIA, form an active MOSFET-C mixer. Because of the high frequency, the MOSFETs can not be used as switches anymore, but instead they are used as variable resistors.

\section{A. Design of the mixing transistors}

The four NMOS mixing transistors perform the actual mixing operation of the $200 \mathrm{GHz}$ LO and RF signals, based on the MOSFET-C mixer principle [1]. They are biased in the linear region $\left(V_{D S}=0\right)$ and are arranged as a double balanced structure. The LO is applied to the drains and the RF is applied to the gates. The gate voltage varies the MOSFET's channel resistance, generating a current which is proportional to the product of the LO and the RF voltages, as can be seen from the well known equation for a MOSFET in the linear region:

$$
i_{D S}=\mu C_{o x} \frac{W}{L}\left[\left(v_{G S}-V_{T}\right) v_{D S}-\frac{v_{D S}^{2}}{2}\right]
$$

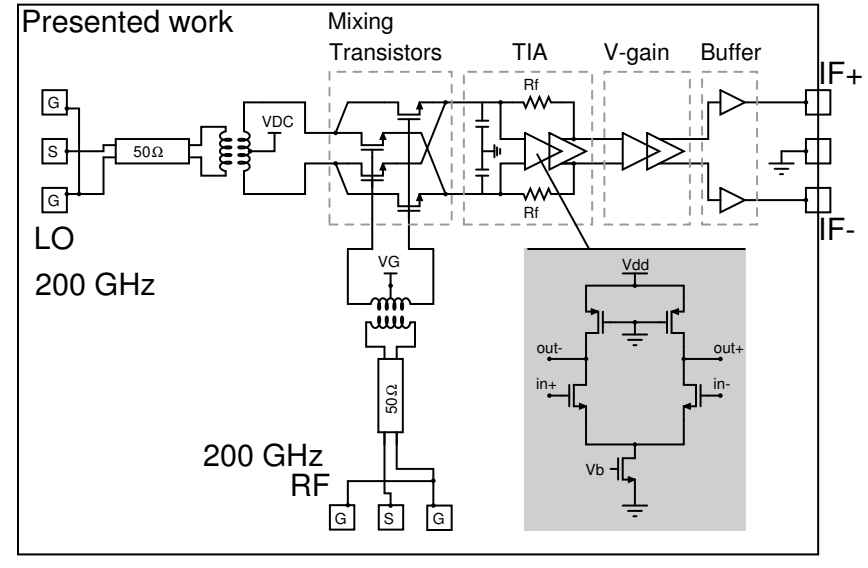

Fig. 1. Circuit diagram of the complete $200 \mathrm{GHz}$ downconverter chip

The double balanced structure cancels unwanted mixing products, while the input capacitance of the TIA (explicitly shown in figure 1) filters out high frequencies so that only the IF signal remains.

The NMOS mixing transistors are laid out in a triple-well structure as to allow the bulk voltage to be set independently from the IF part of the circuit. The bulk voltage is chosen equal to the DC voltage on the drains and sources of the mixing transistors in order to eliminate the bulk effect. This DC voltage is at the same time the bias voltage on the gates of the input transistors of the TIA.

The gate length of the mixing transistors is minimal: $L=$ $90 \mathrm{~nm}$ and the finger width: $W_{F}=1 \mu \mathrm{m}$. The number of fingers is 6 . This determines the impedances presented to the RF and LO transformers to guarantee maximum power transfer to the transistors. The gate-source voltage is set to $V_{G S}=0.38 \mathrm{~V}$ for maximum conversion gain. Thus, the mixing transistors are optimized for maximum available IF power, given the available power of the RF and LO signals. Besides maximizing the conversion gain, this also minimizes the noise figure of the mixing transistors, whose contribution to the total noise figure is most important.

\section{B. Design of the RF and LO transformers}

The entire circuit is fully differential. Two on-chip $200 \mathrm{GHz}$ transformers convert the unbalanced RF and LO signals to balanced signals for the mixing transistors. They match the impedance seen in the RF and LO connections of the mixing 
transistors to $50 \Omega$ at $200 \mathrm{GHz}$ and they are also used for DC bias of the mixing transistors via the center tap of the secondary turn. This way, the gates of the input pair of the TIA are also biased, as mentioned before. Thanks to this approach, complicated bias circuits can be omitted. Both integrated baluns have a single turn in the primary and secondary coil. They are fabricated in the top metal, which is farthest away from the substrate and has a low resistivity. Their trace widths and diameters are optimized toward impedance matching and minimum loss using Momentum.

\section{The IF part}

The function of the TIA is to convert the IF current from the mixing transistors into a voltage. The TIA is designed as a cascade of differential voltage amplifiers, with feedback resistors $R_{f}$, see Fig. 1. Each voltage amplifier has finite gain and bandwidth. Cascading them increases the gain but decreases the bandwidth [2]. The feedback resistors determine the transimpedance and the bandwidth of the TIA. Considering these influences, two voltage amplifiers are cascaded and the feedback resistors are chosen to be $R_{f}=1 \mathrm{k} \Omega$. The voltage gain stage is again a cascade of two of the same voltage amplifiers. The voltage buffers are designed to drive a $50 \Omega$ load.

\section{Layout}

To determine the correct impedances seen at the gates and drains of the mixing transistors and to minimize resistive losses, parasitic extraction is done for the mixing transistors and Momentum simulations are performed for all the surrounding interconnections.

In the IF part, parasitic extraction is done for the TIA, voltage amplifiers and source followers, in order to accurately simulate the performance.

In the chip micrograph (Fig. 9), the transformers can be clearly distinguished. In between them are the mixing transistors and above them is the IF part.

\section{Measurement Results}

The design has been fabricated in a $90 \mathrm{~nm}$ standard CMOS process. The chip is mounted on an FR-4 PCB for measurements. The $200 \mathrm{GHz} \mathrm{LO}$ and RF signals are generated by millimeterwave source modules and applied to the chip through G-band waveguide probes. The IF output is brought off-chip through bondwires. The measurement setup on the probe station is shown in Fig. 2.

Applying the LO with a frequency of $f_{\mathrm{LO}}=200 \mathrm{GHz}$ and a power of $P_{\mathrm{LO}}=-14.9 \mathrm{dBm}$ and the RF with a frequency of $f_{\mathrm{RF}}=200.5 \mathrm{GHz}$ and a power of $P_{\mathrm{RF}}=-16.6 \mathrm{dBm}$ results in a $500 \mathrm{MHz}$ IF with a power of $P_{\mathrm{IF}}=-10.0 \mathrm{dBm}$. The measured conversion gain is $+6.6 \mathrm{~dB}$. As can be seen in Fig. 3, the conversion gain varies linearly with the applied LO power, as is expected for this type of circuit.

Linearity is measured for $P_{\mathrm{LO}}=-14.9 \mathrm{dBm}$, by varying the $\mathrm{RF}$ input power and tracking the first harmonic at $500 \mathrm{MHz}$ $\left(P_{\mathrm{IF}}\right)$ and the third harmonic at $1.5 \mathrm{GHz}\left(P_{3}\right)$. Simulations

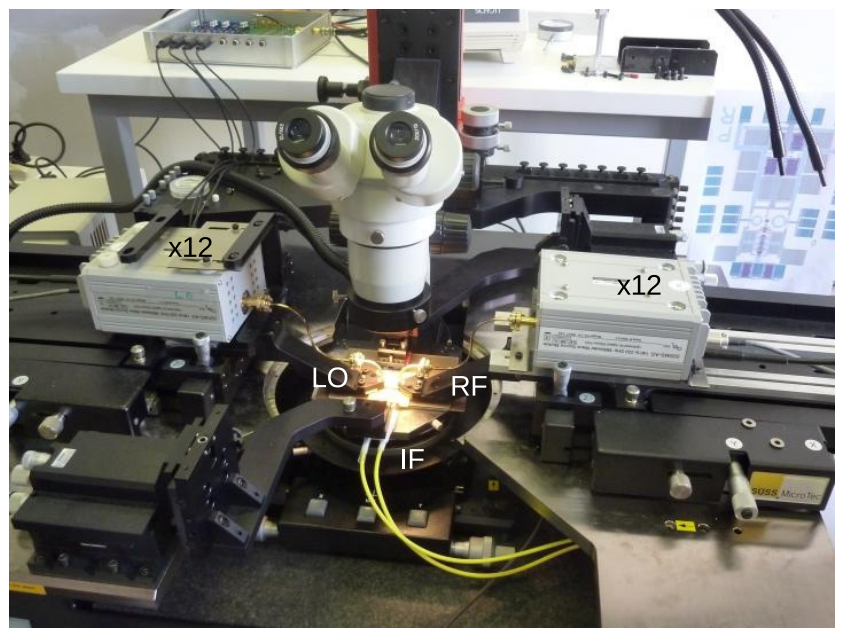

Fig. 2. Photograph of the measurement setup for a $200 \mathrm{GHz}$ downconverter IC, showing the G-band source modules. Signals are applied through probes.

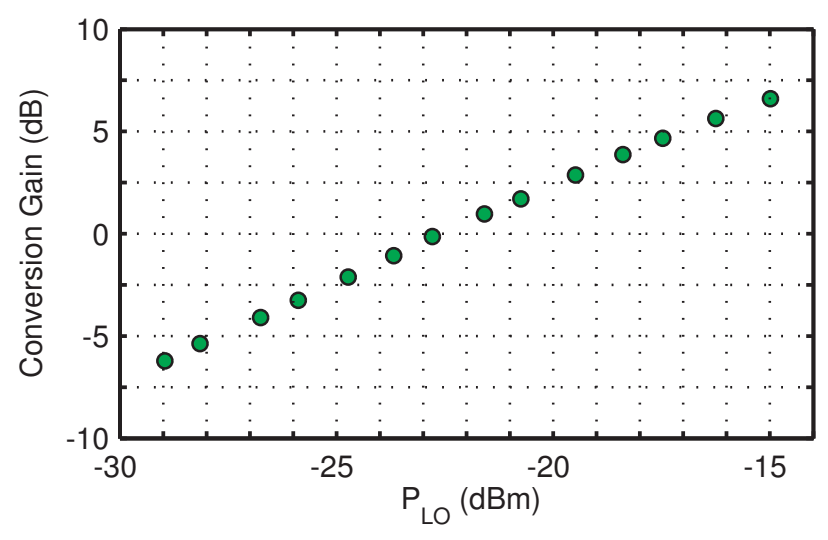

Fig. 3. Measured conversion gain versus $P_{\mathrm{LO}}$.

indicate that the linearity of the complete chip is limited by the IF part only and not by the $200 \mathrm{GHz}$ mixing transistors. Although a true two-tone test could not be performed, the $\mathrm{IP}_{3}$ can be calculated from the theoretical link between the third order intermodulation product $\left(\mathrm{IM}_{3}\right)$ and $P_{3}: \mathrm{IM}_{3}=$ $P_{3}+9.54 \mathrm{~dB}$. As a result, the input third order intermodulation intercept point is: $\operatorname{IIP}_{3}=-5.4 \mathrm{dBm}$. This corresponds very well to the simulations, as shown in Fig. 4.

The IF output bandwidth is measured by changing $f_{\mathrm{RF}}$ while keeping $f_{\mathrm{LO}}$ fixed at $200 \mathrm{GHz}$. As can be seen in Fig. 5, the IF bandwidth is $3 \mathrm{GHz}$. This is limited by the IF gain stages but also by the bondwires and the FR-4 PCB.

The conversion gain deviates less than $\pm 1.5 \mathrm{~dB}$ within an $\mathrm{LO}$ and RF frequency range of $26 \mathrm{GHz}$ : from 186 to $212 \mathrm{GHz}$. This is measured by changing $f_{\mathrm{RF}}$ and $f_{\mathrm{LO}}$ simultaneously while keeping $f_{\mathrm{IF}}$ fixed at $500 \mathrm{MHz}$. This downconverter can be used for carrier frequencies anywhere between 186 and $212 \mathrm{GHz}$. The reason is that the transformers match the mixing transistors with $50 \Omega$ in a very wide band.

The output noise measurements and simulations are shown 


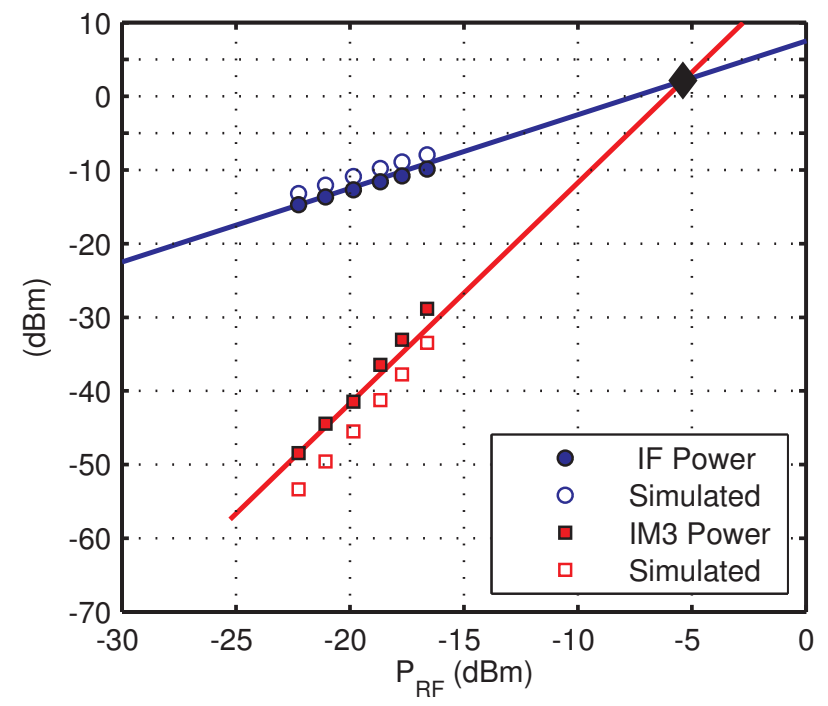

Fig. 4. Measured and simulated $P_{\mathrm{IF}}$ and $\mathrm{IM}_{3}$ versus $P_{\mathrm{RF}}$.

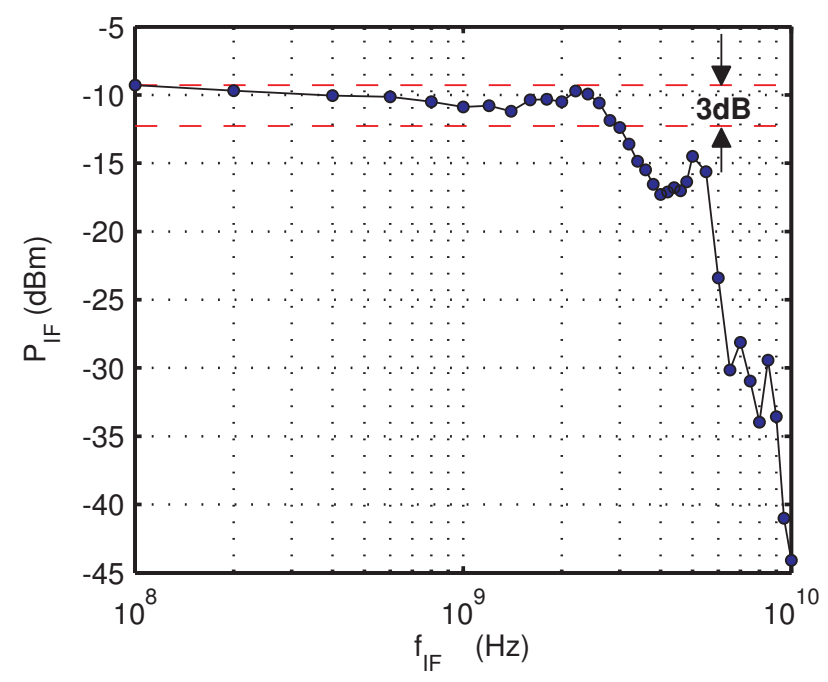

Fig. 5. Measured IF bandwidth is $3 \mathrm{GHz}$.

in Fig. 6. The average noise level in a band from $10 \mathrm{kHz}$ to $3 \mathrm{GHz}$ is $-137.5 \mathrm{dBm} / \mathrm{Hz}$. The $1 / \mathrm{f}$ noise corner frequency is around $10 \mathrm{MHz}$. Compared to the $3 \mathrm{GHz}$ bandwidth, the 1/f noise can be neglected. From the integrated noise, the noise figure can be calculated:

$$
\mathrm{NF}=10 \times \log \left(\frac{N_{o}}{G k T B}\right)=29.9 \mathrm{~dB}
$$

Where $N_{o}$ is the integrated output noise power in the band, calculated from the measured data. $G$ is the conversion gain and $k T B$ is the available input noise power. This is a single sideband (SSB) noise figure since it assumes a signal on one side of the LO frequency only.

As a demonstration of the IC's high-speed data communication capability at millimeterwave frequencies, a test setup was conceived to generate $200 \mathrm{GHz}$ BPSK and QPSK modulated

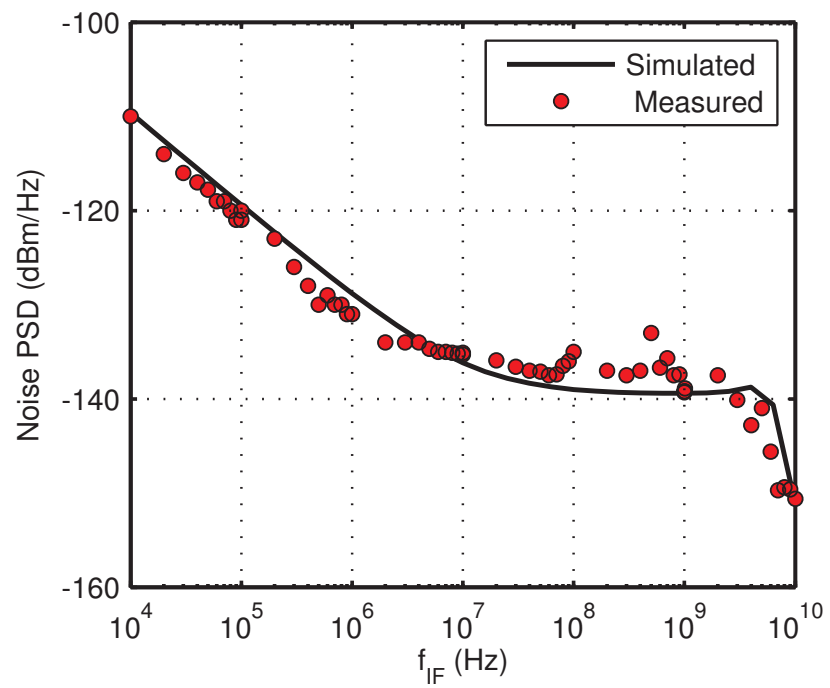

Fig. 6. Measured and simulated output noise density. 1/f noise corner around $10 \mathrm{MHz}$, average noise level in a band from $10 \mathrm{kHz}$ to $3 \mathrm{GHz}$ : $-137.5 \mathrm{dBm} / \mathrm{Hz}$

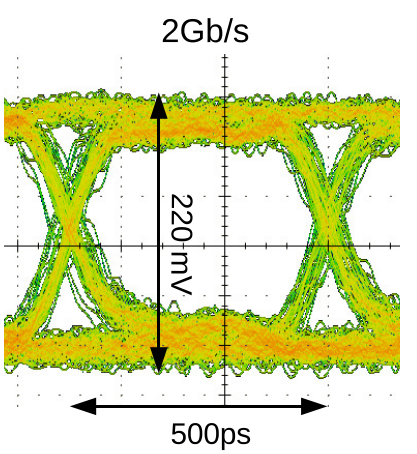

(a)

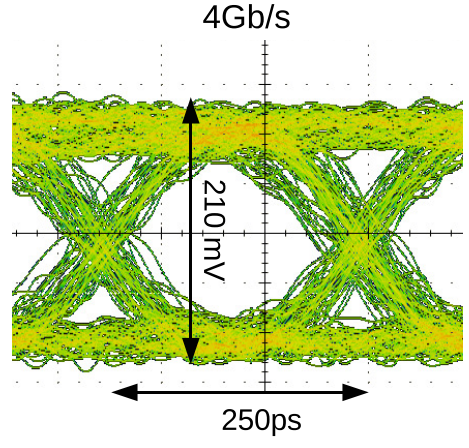

(b)
Fig. 7. Measured eye diagrams for BPSK with data rates of 2 Gbit/s (a) and $4 \mathrm{Gbit} / \mathrm{s}$ (b), downconverted to baseband.

signals to apply to the RF input. The millimeter wave source module multiplies any phase or frequency information at its input with a factor of twelve. Therefore, signals are first generated that, when applied to the source module's input, give the desired BPSK or QPSK signals at the output. By choosing the LO frequency equal to the carrier frequency of the modulated signals, the presented IC mixes the signal to baseband. The output of the downconverter can be visualized on an oscilloscope in the form of eye diagrams. In Fig. 7, eye diagrams for $2 \mathrm{Gbit} / \mathrm{s}$ and $4 \mathrm{Gbit} / \mathrm{s}$ BPSK are shown.

Fig. 8 shows the measured constellation diagrams for BPSK and QPSK signals downconverted to different IF frequencies for various data rates.

It is thus shown that the presented downconverter IC is capable of achieving high data rates at G-band frequencies. It should be noted that the speed is limited by the IF part and the FR-4 PCB and not by the $200 \mathrm{GHz}$ mixing transistors. The speed can be easily enhanced by optimizing the IF blocks. 


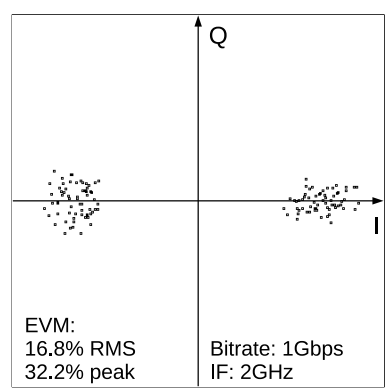

(a)

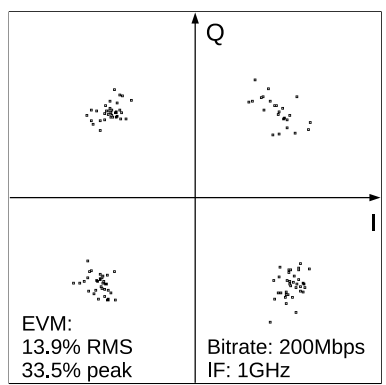

(c)

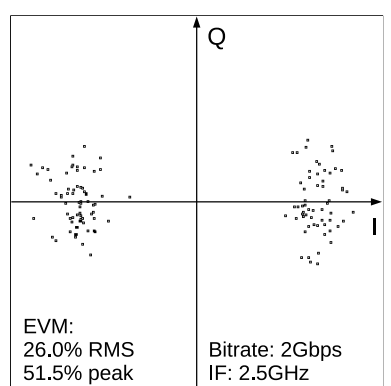

(b)

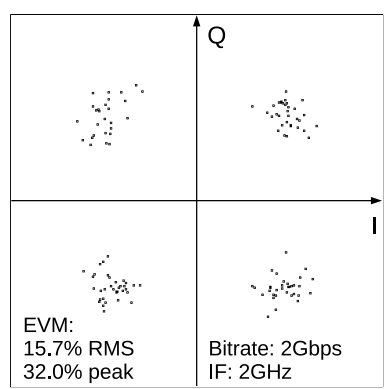

(d)
Fig. 8. Measured downconverted constellation diagrams for BPSK with data rates of $1 \mathrm{Gbit} / \mathrm{s}$ (a) and $2 \mathrm{Gbit} / \mathrm{s}$ (b) and QPSK with data rates of $200 \mathrm{Mbit} / \mathrm{s}$ (c) and $2 \mathrm{Gbit} / \mathrm{s}$ (d), at different IF frequencies.

The circuit draws $53 \mathrm{~mA}$ from a $1.2 \mathrm{~V}$ supply voltage. This power is consumed in the TIA, the voltage gain stage and the buffer stage.

A die photograph is shown in Fig. 9. The chip has an area of $0.375 \mathrm{~mm}^{2}$, this includes the whole circuit, bond pads and probe pads. The active area as indicated in Fig. 9 is only $0.04 \mathrm{~mm}$.

\section{CONCLUSION}

In table I, the performance of this chip is summarized and compared to other work. The presented work is the only downconverter at these high frequencies with both positive conversion gain and considerable IF bandwidth. Moreover, the required LO power is relatively low, which is an important consideration in this frequency region. To the author's knowledge, this circuit is also the only one capable of downconverting modulated signals with high data rates, at carrier frequencies above $f_{\max }$.

\section{ACKNOWLEDGMENT}

This research is partly supported by the ERC Advanced Grant 227680 (DARWIN).

\section{REFERENCES}

[1] J. Crols and M. Steyaert, "A 1.5 GHz highly linear CMOS downconversion mixer," Solid-State Circuits, IEEE Journal of, vol. 30, no. 7, pp. 736 -742, July 1995.

[2] F. Tavernier and M. Steyaert, "High-speed optical receivers with integrated photodiode in $130 \mathrm{~nm}$ CMOS," Solid-State Circuits, IEEE Journal of, vol. 44, no. 10, pp. 2856 -2867, 2009.

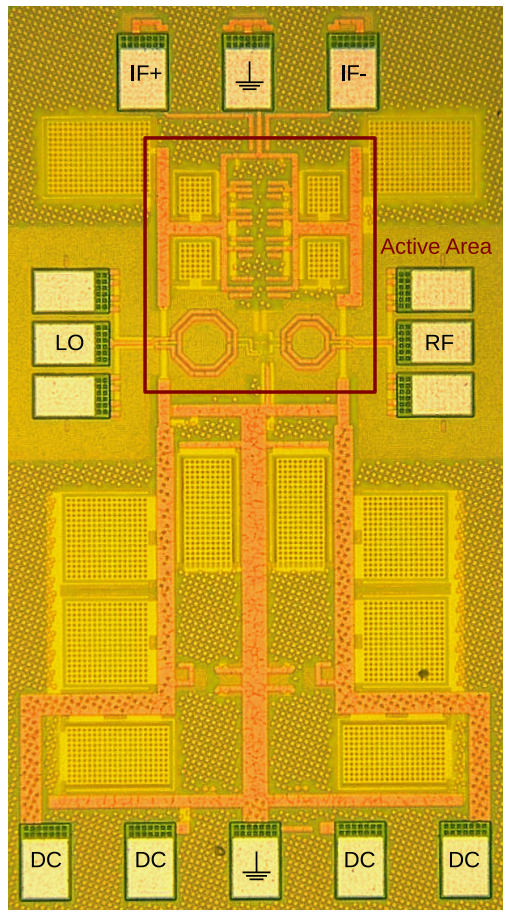

Fig. 9. Chip micrograph with indication of the active area

TABLE I

PERFORMANCE SUMMARY AND COMPARISON TO OTHER WORK

\begin{tabular}{|l|c|c|c|c|}
\hline Reference & {$[3]$} & {$[4]$} & {$[5]$} & $\begin{array}{c}\text { This } \\
\text { work }\end{array}$ \\
\hline Process & $\begin{array}{c}0.1 \mu m \text { GaAs } \\
\text { mHEMT }\end{array}$ & $\begin{array}{c}65 \mathrm{~nm} \\
\text { CMOS }\end{array}$ & $\begin{array}{c}0.25 \mu \mathrm{m} \\
\text { CMOS }\end{array}$ & $\begin{array}{c}90 \mathrm{~nm} \\
\text { CMOS }\end{array}$ \\
\hline RF $(\mathrm{GHz})$ & 220 & 102 & 650 & 200 \\
\hline IF $(\mathrm{MHz})$ & 2000 & 2000 & 0 to 1.6 & $\begin{array}{c}0 \text { to } \\
3000\end{array}$ \\
\hline Conv. Gain $(\mathrm{dB})$ & -12 & -4 & - & +6.6 \\
\hline IIP $3(\mathrm{dBm})$ & - & - & - & -5.4 \\
\hline NF $(\mathrm{dB})$ & $8.4 \mathrm{DSB}$ & 22 & $68 \mathrm{SSB}$ & $29.9 \mathrm{SSB}$ \\
\hline$P_{\mathrm{LO}}(\mathrm{dBm})$ & - & +1 & -32.5 & -14.9 \\
\hline$P_{\mathrm{DC}}(\mathrm{mW})$ & - & - & - & 63.3 \\
\hline Area $\left(\mathrm{mm}{ }^{2}\right)$ & 3 & - & - & 0.375 \\
\hline Data rate $(\mathrm{Gbit} / \mathrm{s})$ & - & - & - & $>4$ \\
\hline
\end{tabular}

[3] S. Gunnarsson, N. Wadefalk, J. Svedin, S. Cherednichenko, I. Angelov, H. Zirath, I. Kallfass, and A. Leuther, "A $220 \mathrm{GHz}$ single-chip receiver MMIC with integrated antenna," Microwave and Wireless Components Letters, IEEE, vol. 18, no. 4, pp. 284 -286, 2008.

[4] S. Nicolson, A. Tomkins, K. Tang, A. Cathelin, D. Belot, and S. Voinigescu, "A $1.2 \mathrm{v}, 140 \mathrm{GHz}$ receiver with on-die antenna in $65 \mathrm{~nm}$ CMOS," in Radio Frequency Integrated Circuits Symposium, 2008. RFIC 2008. IEEE, 172008.

[5] U. Pfeiffer, E. Ojefors, A. Lisaukas, D. Glaab, and H. Roskos, "A CMOS focal-plane array for heterodyne terahertz imaging," in Radio Frequency Integrated Circuits Symposium, 2009. RFIC 2009. IEEE, 2009, pp. 433 -436 . 